\title{
Utilização de fármacos vencidos no estado sólido e líquido como inibidores de corrosão em materiais metálicos
}

\section{Use of drugs in the solid and liquid state as inhibitors of corrosion in metallic materials}

\section{Uso de fármacos caducados en estado sólido y líquido como inhibidores de corrosión en materiales metálicos}

\section{Utilisation de médicaments solides et liquides comme inhibiteurs de corrosion dans les matériaux métalliques}

Matheus da Silva Lages iD matheuslages@yahoo.com.br Universidade Federal dos Vales do Jequitinhonha e Mucuri (UFVJM)

Carlos Alexandre Teixeira de Matos iD

carlosalexandretmatos@gmail.com Universidade Federal dos Vales do Jequitinhonha e Mucuri (UFVJM)

Cristiano Batista Rodrigues (iD cristianocvk@gmail.com Universidade Federal dos Vales do Jequitinhonha e Mucuri (UFVJM)

Ellen Pires Saigg iD ellen.saigg@gmail.com Universidade Federal dos Vales do Jequitinhonha e Mucuri (UFVJM)

Karine Silva iD

karinemnovas@gmail.com Universidade Federal dos Vales do Jequitinhonha e Mucuri (UFVJM)

\section{Resumo}

A corrosão metálica é o ataque destrutivo e não intencional de um metal. Esse ataque começa, normalmente, pela superfície. Na indústria, a corrosão metálica é um grande problema, pois causa danos aos materiais metálicos. Por se tratar de um processo irreversível, torna-se necessário buscar alternativas para eliminar (ou reduzir) esse tipo de corrosão. Este artigo tem como finalidade estudar a eficiência de fármacos vencidos no estado sólido (apresolina, cloridrato de tipoclidina, digoxina e pilem levonorgestrel) e no estado líquido (dipirona monoidratada, maleato de timolol e nistatina). Entre os fármacos sólidos, o cloridrato de tipoclidina se destacou ao apresentar bons valores de eficiência contra a corrosão, sendo observada uma eficiência de $66,3 \%$. Já entre os fármacos líquidos, a dipirona e a nistatina apresentaram eficiência de 59,4\% e 61,5\%, respectivamente, no combate a corrosão.

Palavras-chaves: Inibidores de corrosão. Corrosão metálica. Taxa de corrosão.

\begin{abstract}
Metal corrosion is the destructive and unintentional attack of metal and normally begins on the surface. In the industry, metal corrosion is a huge problem as it causes damage to metal materials. As it is an irreversible process, it is necessary to search for alternatives to eliminate this type of corrosion. This article aims to study the efficiency of overdue solid-state (Apresoline, Tipoclidine Hydrochloride, Digoxinand Pilem Levonorgestrel) and in a liquid state (Dipirone Monohydrate, Timolol Maleate, and Nystatin). Among solid drugs, Tipoclidine Hydrochloride has been highlighted when presenting good efficiency against the corrosion, being observed efficiency of $66,3 \%$. Liquid drugs, as Dipirone and Nystatin provide efficiency of $59,4 \%$ and $61,6 \%$, respectively, in the fight against corrosion.
\end{abstract}

Keywords: Corrosion inhibitors. Metal corrosion. Corrosion rate.

\section{Resumen}

La corrosión metálica es el ataque destructivo y no intencional de un metal; este ataque empieza normalmente por la superficie. En la industria, la corrosión metálica es un gran problema porque causa daños a los materiales metálicos. Por tratarse de un proceso irreversible, se hace necesario buscar alternativas para eliminar (o reducir) este tipo de corrosión. Este trabajo tiene como objetivo estudiar la eficiencia de fármacos caducados en el estado sólido (Apresolina, Cloridrato de Tipoclidina, Digoxina y Pilem Levonorgestrel) y en el estado líquido (Dipirona Monoidratada, Maleato de Tomolol y Nistatina). Entre los 
fármacos sólidos, el Cloridrato de Tipoclidina se destacó al presentar valores buenos de eficiencia contra la corrosión, siendo observada una eficiencia de 66,3\%. Entre los fármacos líquidos, la Dipirona y la Nistatina presentaron eficiencia de $59,4 \%$ y $61,5 \%$, respectivamente, en el combate a la corrosión.

Palabras claves: Inhibidores de corrosión. Corrosión metálica. Tasa de corrosión.

\section{Résumé}

La corrosion des métaux s'agit d'une attaque destructive et involontaire d'un métal; Cette attaque commence généralement à la surface. Dans l'industrie, la corrosion des métaux est un problème majeur car elle endommage les matériaux métalliques. Comme il s'agit d'un procédé irréversible, il est nécessaire de rechercher des alternatives pour éliminer (ou réduire) ce type de corrosion. Cet article a pour objectif d'étudier l'efficacité des médicaments périmés à l'état solide (Apresoline, Chlorhydrate de Tipoclidine, Digoxine et Levonorgestrel) et à l'état liquide (Dipyrone Monohydrate, Timolol Maleate et Nystatine). Parmi les médicaments solides, le chlorhydrate de triprolidine se distingue par sa bonne efficacité contre la corrosion: c'est à dire, une efficacité de $66,3 \%$. Parmi les médicaments liquides, le Dipirone et la Nystatine ont montré une efficacité de $59,4 \%$ et $61,5 \%$, respectivement, dans la lutte contre la corrosion.

Mots clés: Inhibiteurs de corrosion. Corrosion métallique. Taux de corrosion.

\section{Introdução}

A corrosão metálica é o processo de deterioração de um metal por ação eletroquímica com o meio em que se encontra, sendo um processo espontâneo e irreversível, no qual o material metálico passa do estado reduzido para oxidado. Num cenário industrial, a corrosão pode acarretar problemas, como danos materiais e custos de reparo e substituição de peças. Estima-se que cerca de $5 \%$ dos gastos de uma nação industrializada estão relacionados com a prevenção da corrosão, a manutenção ou substituição de peças perdidas ou comprometidas pelo processo de corrosão (CALLISTER, 2008). Os custos evitáveis giram em torno de U 5 bilhões ao ano.

Alguns métodos são utilizados para prevenir a corrosão, como o uso de revestimentos protetores, substituição do material susceptível à corrosão por outros mais resistentes, proteção catódica, uso de inibidores e, ainda, outros mais recentes, como a aplicação de métodos elétricos para avaliar revestimentos orgânicos em aço (KENDIG; SCULLY, 1990) e a capacidade da química quântica de selecionar inibidores de corrosão pelos métodos de impedância, potenciostático e polarização (Ll et al., 1999).

Os inibidores de corrosão são substâncias que, em concentração correta, são capazes de eliminar ou diminuir o processo de corrosão em um material. Os inibidores atuam sendo absorvidos pela superfície metálica, criando uma película que diminui a taxa corrosão devido ao abrandamento das reações anódicas, catódicas ou ambas (FRAUCHES-SANTOS et al., 2014).

Compreender os aspectos químicos, físicos e mecânicos dos materiais e os diversos efeitos que incidem sobre eles são de vital importância, uma vez que, encontrando as relações corretas de causa-efeito, é possível encontrar uma maneira de retardar ou acelerar esses efeitos. São várias as utilizações dos inibidores nos mais variados segmentos industriais atuais, mas podemos destacar: a construção civil (ataque nas estruturas de aço), a aviação (proteção nas aeronaves), o setor de autopeças (preservação da parte externa de seus componentes) (ABRACO, 2007) e a indústria de petróleo e gás, em que são feitos estudos do comportamento corrosivo de aços inoxidáveis nas refinarias, pois, devido às impurezas encontradas no petróleo, apresentam um elevado grau de corrosão (NASCIMENTO et al., 2004).

A utilização de fármacos como inibidores de corrosão foi estudada por Fernandes et al. (2017) e por Paranhos et al. (2017), que concluíram que alguns fármacos atuam eficientemente contra a corrosão. 
Alguns fármacos estudados pelos pesquisadores apresentaram elevada eficiência contra a corrosão, como a fluormetolona, a benzocaína, a metoclopramida e o triazol. Desse modo, torna-se interessante a continuação do estudo de fármacos como inibidores de corrosão.

A utilização de fármacos vencidos contra a corrosão surge como uma alternativa limpa e econômica para tratar esse processo que resulta em grandes problemas para a indústria. Diante disso, este estudo teve como objetivo avaliar a eficiência dos fármacos apresolina, cloridrato de tipoclidina, digoxina, pilem levonorgestre, dipirona monoidratada, maleato de timolol e nistatina, quando vencidos, em diferentes concentrações, para verificar a eficiência deles como inibidores de corrosão em material metálico.

A verificação da eficiência ou não de um fármaco contra a corrosão parte da análise da taxa de corrosão, que é uma metodologia que expõe o desgaste ocorrido na superfície do material como consequência da corrosão e, desse modo, a taxa de corrosão é de fundamental importância no controle da corrosão. É possível quantificar a taxa de corrosão de diversas maneiras, sendo a mais comum o cálculo da taxa de penetração de corrosão (TPC), que relaciona a perda de material por unidade de tempo. Como há uma corrente elétrica associada às reações de corrosão eletroquímica, também é possível expressar a taxa em função da densidade de corrente (corrente por unidade de área superficial de material corroendo) (MORA; SIHVENGER, 2006).

A eficiência do fármaco como inibidor de corrosão será calculada através da TPC Eq.(1) do material exposto ao ambiente corrosivo, com e sem inibidor (CALLISTER, 2008):

$$
T P C=\frac{K \times W}{A \times \rho \times t}
$$

W: a variação de massa ou a perda de massa $(\mathrm{mg})$;

A: a área da superfície de contato do corpo de prova com o ambiente corrosivo $\left(\mathrm{cm}^{2}\right)$;

$\rho:$ a massa específica do material metálico $\left(\mathrm{g} / \mathrm{cm}^{3}\right)$;

$t$ : tempo do ensaio (horas);

K: constante que depende da unidade de medida desejada para a TPC. Para uma TPC fornecida em $\mathrm{mm} / \mathrm{ano}$, o valor de $\mathrm{K}$ deve ser igual a 87,6 .

A eficiência dos inibidores pode ser estimada através dos valores da TPC considerando ensaios em branco (sem inibidor) e os valores obtidos da TPC com inibidores Eq.(2):

$$
n(\%)=\left|\frac{T_{C}-T_{C} \text { inib }}{T_{C}}\right| \times 100
$$

Onde:

$n$ é a eficiência do inibidor (em porcentagem);

Tc é a taxa de penetração de corrosão calculada para o ensaio em branco;

$T_{C}$ inib é a taxa de penetração de corrosão com uso do inibidor.

Neste trabalho, realizaram-se experimentos com alguns fármacos vencidos para estimar os seus efeitos de retardamento (ou não) no processo corrosivo. Conforme os resultados apresentados por Cerqueira et al. (2018), a análise da eficiência de cada fármaco foi realizada utilizando materiais de aço carbono como o corpo de prova a ser submetido à corrosão.

\section{Metodologia}

Porcas de aço carbono foram preparadas para serem utilizadas como corpos de prova. Primeiramente, realizou-se o lixamento dos corpos de prova, com lixa d'água (granulometria 400), para retirada de defeitos do material, eliminando as irregularidades na superfície das porcas. Posteriormente, aferiram-se as dimensões dos corpos de prova usando um paquímetro Fig.(1-A). Então, limparam-se quimicamente os corpos de prova com escova, água, surfactante, etanol e acetona, respectivamente Fig.(1-B). 
Figura 1 - Preparação dos corpos de prova: medição das dimensões (A) e limpeza química (B).

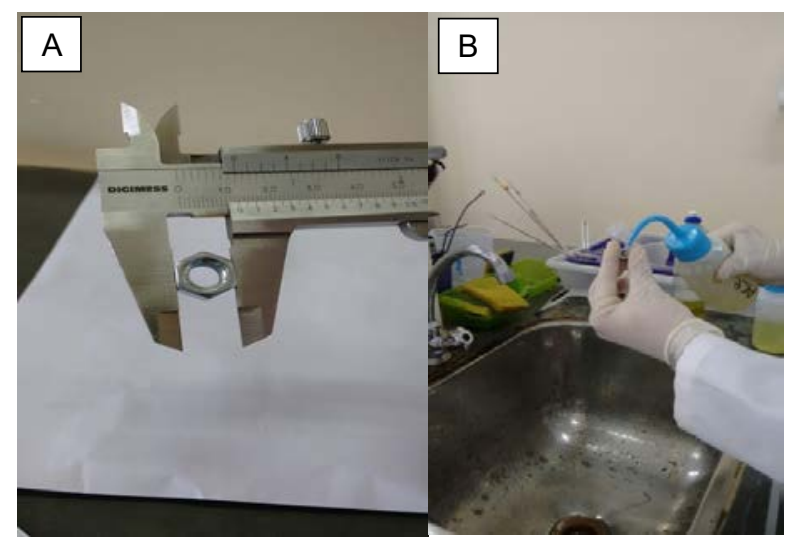

Fonte: Elaboração própria (2019).

Após a limpeza os corpos de prova, foram secados e pesados em uma balança analítica Fig.2. Paralelamente, foram inseridos em tubos de ensaios $10 \mathrm{ml}$ de ácido clorídrico puro $(\mathrm{HCl})$, sendo inseridos os fármacos em quantidades estipuladas. As Tabelas 1 e 2 mostram as concentrações dos fármacos sólidos e líquidos, respectivamente.

Figura 2 - Secagem (A) e pesagem (B) dos corpos de prova.

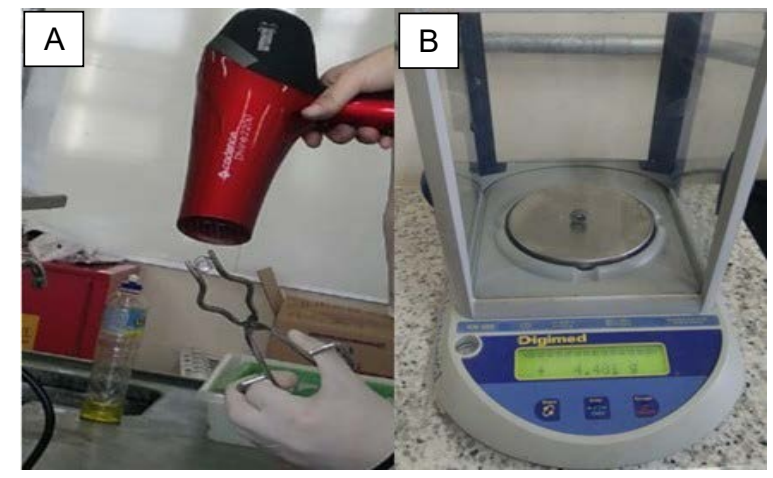

Fonte: Elaboração própria (2019).

Tabela 1 - Condições experimentais para fármacos sólidos.

\begin{tabular}{c|c|c}
\hline Fármacos & Condição experimental & Concentração (mg) \\
\hline \multirow{4}{*}{ Apresolina } & 01 & 25,0 \\
\cline { 2 - 3 } & 02 & 50,0 \\
\cline { 2 - 3 } & 03 & 75,0 \\
\cline { 2 - 3 } & 04 & 100,0 \\
\hline \multirow{3}{*}{ Cloridrato de tipoclidina } & 05 & 250,0 \\
\cline { 2 - 3 } & 06 & 500,0 \\
\cline { 2 - 3 } & 07 & 750,0 \\
\hline \multirow{3}{*}{ Digoxina } & 08 & 0,25 \\
\cline { 2 - 3 } & 09 & 1,0 \\
\cline { 2 - 3 } & 10 & 2,0 \\
\cline { 2 - 3 } & 11 & 3,0 \\
\hline \multirow{2}{*}{ Pilem levonorgestrel } & 12 & 0,75 \\
\cline { 2 - 3 } & 13 & 2,25 \\
\hline
\end{tabular}

Fonte: Elaboração própria (2019). 
Tabela 2 - Condições experimentais para fármacos líquidos.

\begin{tabular}{|c|c|c|c|}
\hline Fármacos & Condição experimental & Concentrações (ml) & Observações \\
\hline \multirow{6}{*}{ Dipirona monoidratada } & 01 & 1,0 & - \\
\hline & 02 & 2,0 & - \\
\hline & 03 & 3,0 & - \\
\hline & 04 & 4,0 & - \\
\hline & 05 & 6,0 & - \\
\hline & 06 & 8,0 & - \\
\hline \multirow{4}{*}{ Maleato de timolol } & 07 & 1,0 & - \\
\hline & 08 & 2,0 & - \\
\hline & 09 & 3,0 & - \\
\hline & 10 & 4,0 & - \\
\hline \multirow{4}{*}{ Nistatina } & 11 & 1,0 & - \\
\hline & 12 & 2,0 & - \\
\hline & 13 & 3,0 & - \\
\hline & 14 & 4,0 & - \\
\hline- & 15 & - & Ensaio em branco \\
\hline
\end{tabular}

Fonte: Elaboração própria (2019).

Os corpos de prova foram inseridos nos tubos contendo o ácido e os fármacos, permanecendo em repouso durante 5 horas no meio corrosivo Fig.3. Também foi realizado um ensaio em branco, ou seja, sem o uso de fármacos. Após esse tempo, as porcas foram retiradas dos tubos de ensaio para sua posterior limpeza, usando os mesmos elementos da primeira limpeza. Por fim, os corpos de prova foram secados e pesados para o cálculo de TCP e eficiência.

Figura 3 - Corpos de prova em ambiente ácido.

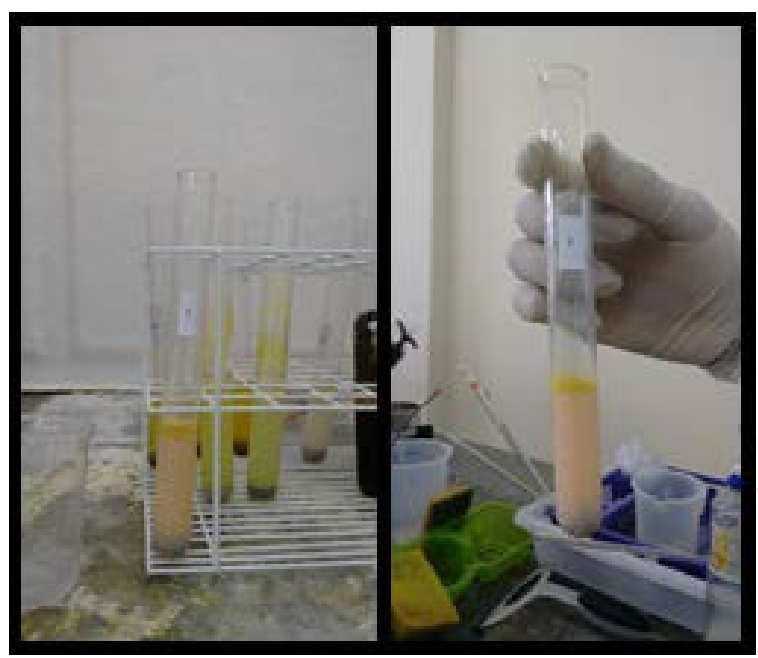

Fonte: Elaboração própria (2019). 


\section{Resultados e discussão}

São apresentados, a seguir, os resultados obtidos com suas devidas considerações, separando os fármacos em seus devidos estados: sólidos e líquidos.

\subsection{Análise dos fármacos no estado sólido}

Os resultados obtidos para os fármacos em estado sólido são apresentados na Tab.(3).

Tabela 3 - Taxa de corrosão e eficiência para fármacos vencidos no estado sólido.

\begin{tabular}{c|c|c|c}
\hline \multirow{4}{*}{ Fármacos } & Concentração $(\mathrm{mg})$ & Taxa de corrosão (mm/ano) & Eficiência (\%) \\
\hline \multirow{4}{*}{ Apresolina } & 25,0 & 71,6138 & 1,00 \\
\cline { 2 - 4 } & 50,0 & 73,9064 & $-2,17$ \\
\cline { 2 - 4 } & 75,0 & 68,8605 & 4,81 \\
\hline \multirow{4}{*}{ Cloridrato de tipoclidina } & 100,0 & 69,6130 & 3,77 \\
\cline { 2 - 4 } & 250,0 & 66,3626 & 8,26 \\
\cline { 2 - 4 } & 500,0 & 42,5143 & 41,23 \\
\hline \multirow{4}{*}{ Digoxina } & 750,0 & 24,3763 & 66,30 \\
\cline { 2 - 4 } & 0,25 & 86,0940 & $-19,02$ \\
\cline { 2 - 4 } & 1,0 & 55,7725 & 22,90 \\
\cline { 2 - 4 } & 2,0 & 97,0451 & $-34,16$ \\
\hline \multirow{3}{*}{ Pilem levonorgestrel } & 3,0 & 54,6940 & 24,39 \\
\cline { 2 - 4 } & 0,75 & 72,1239 & 0,30 \\
\hline \multirow{2}{*}{-} & 2,25 & 71,2903 & 1,45 \\
\hline
\end{tabular}

Fonte: Elaboração própria (2019).

Pelos resultados apresentados, observa-se que a apresolina e o pilem levonorgestrel apresentaram efeito neutro contra a corrosão, de modo que não podem ser considerados inibidores de corrosão. O cloridrato de tipoclidina (CLORIDRATO DE TIPOCLIDINA, 2013) apresentou os melhores resultados, pois, à medida que a quantidade do fármaco aumenta, existe uma tendência de aumentar a eficiência contra a corrosão metálica, sendo observado uma eficiência $66,30 \%$ com o uso de $750 \mathrm{mg}$ do fármaco.

A digoxina (DIGOXINA, 2016) apresenta resultados interessantes, uma vez que o fármaco apresenta efeitos positivos contra a corrosão para algumas concentrações (1,0 e 3,0 mg). Entretanto, para outras, apresenta efeito contrário $(0,25$ e 2,0 mg). A resposta dessa característica apresentada pela digoxina pode ser indicada pelo processo de passivação, no qual, em certas quantidades, o material pode atuar inibindo a corrosão e, em outras quantidades e condições, o mesmo material pode atuar acelerando o processo de corrosão. Esse processo de passivação assemelha-se ao processo de passivação do alumínio, que é altamente resistente à corrosão em muitos ambientes, no entanto, uma alteração na natureza do ambiente pode fazer com que o material passivado se reverta para um estado ativo (CALLISTER, 2008). A eficiência da digoxina e dos demais fármacos sólidos é apresentada mais claramente na Fig.4. 
Figura 4 - Eficiência a inibição da corrosão, com o uso de fármacos sólidos.

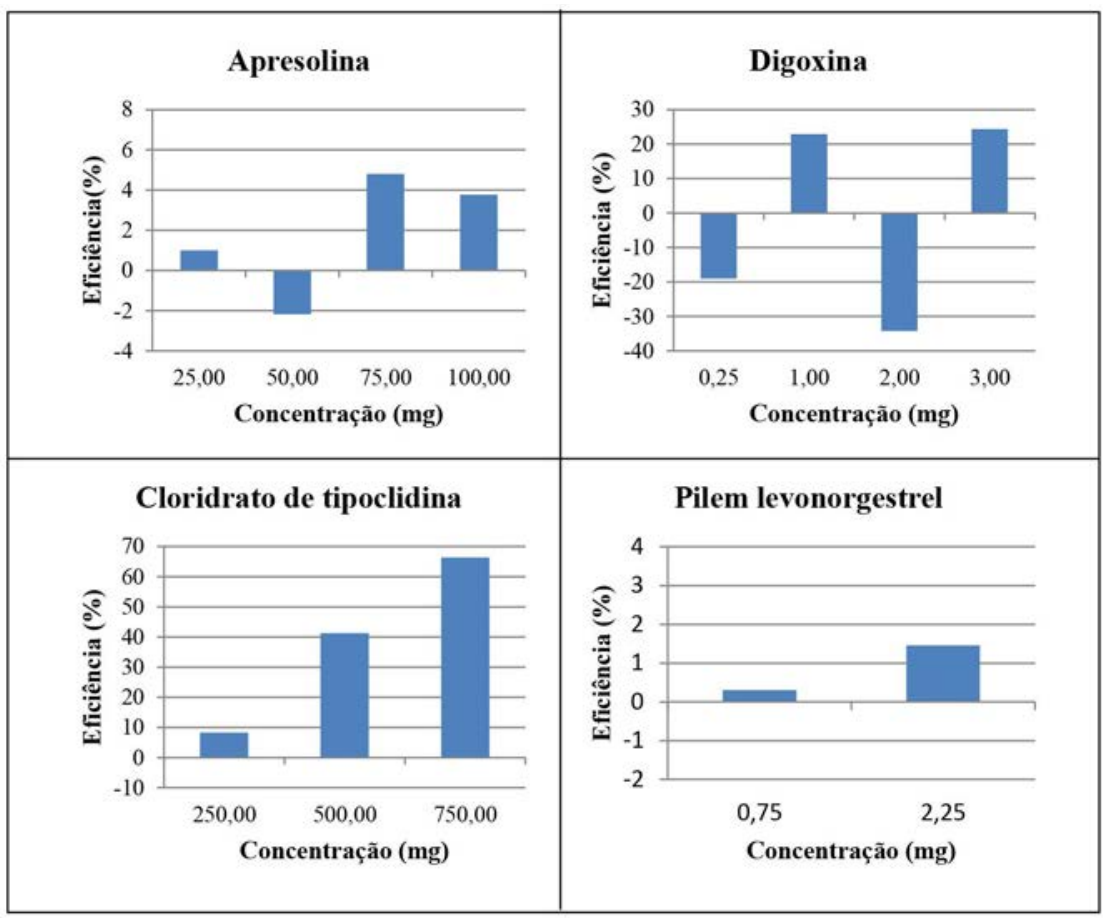

Fonte: Elaboração própria (2019).

\section{Análise dos fármacos no estado líquido}

$\mathrm{Na}$ análise da inibição de corrosão com uso de fármacos líquidos, os corpos de prova, assim como no ensaio com fármacos sólidos, apresentaram corrosão do tipo uniforme, uma vez que o ataque eletroquímico aconteceu de maneira uniforme ao longo de toda a superfície da amostra. Os resultados são apresentados na Tab.(4).

Tabela 4 - Taxa de corrosão e eficiência para fármacos vencidos no estado líquido.

\begin{tabular}{c|c|c|c}
\hline Fármacos & Concentrações $(\mathrm{ml})$ & Taxas de Corrosão $(\mathrm{mm} / \mathrm{ano})$ & Eficiência $(\%)$ \\
\hline \multirow{4}{*}{ Dipirona monoidratada } & 1,0 & 77,017 & $-6,47$ \\
\cline { 2 - 4 } & 2,0 & 30,024 & 58,49 \\
\cline { 2 - 4 } & 3,0 & 29,330 & 59,45 \\
\cline { 2 - 4 } & 4,0 & 29,488 & 59,24 \\
\cline { 2 - 4 } & 6,0 & 70,202 & 2,95 \\
\hline \multirow{4}{*}{ Maleato de timolol } & 8,0 & 51,142 & $-43,54$ \\
\cline { 2 - 4 } & 1,0 & 103,832 & $-225,53$ \\
\cline { 2 - 4 } & 2,0 & 235,484 & $-247,64$ \\
\cline { 2 - 4 } & 3,0 & 251,478 & 247,87 \\
\hline \multirow{3}{*}{ Nistatina } & 4,0 & 251,640 & 32,87 \\
\cline { 2 - 4 } & 1,0 & 53,223 & 61,56 \\
\cline { 2 - 4 } & 2,0 & 48,556 & 58,01 \\
\cline { 2 - 4 } & 3,0 & 27,810 & 0,00 \\
\hline
\end{tabular}

Fonte: Elaboração própria (2019). 
Pode-se observar que os fármacos dipirona monoidratada e nistatina apresentaram resultados promissores, alcançando uma eficiência próxima de $60 \%$ em certas concentrações. O maleato de timolol teve resposta adversa, acelerando o processo de corrosão, o que resultou em uma ineficiência que chega a ser superior a $200 \%$. Desse modo, podemos considerar a dipirona monoidratada e a nistatina como inibidores de corrosão, entretanto essa consideração não é válida para o maleato de timolol. A Figura 5 apresenta de maneira mais clara a eficiência dos fármacos líquidos em cada concentração utilizada.

Figura 5 - Eficiência a inibição da corrosão, com o uso de fármacos líquidos.

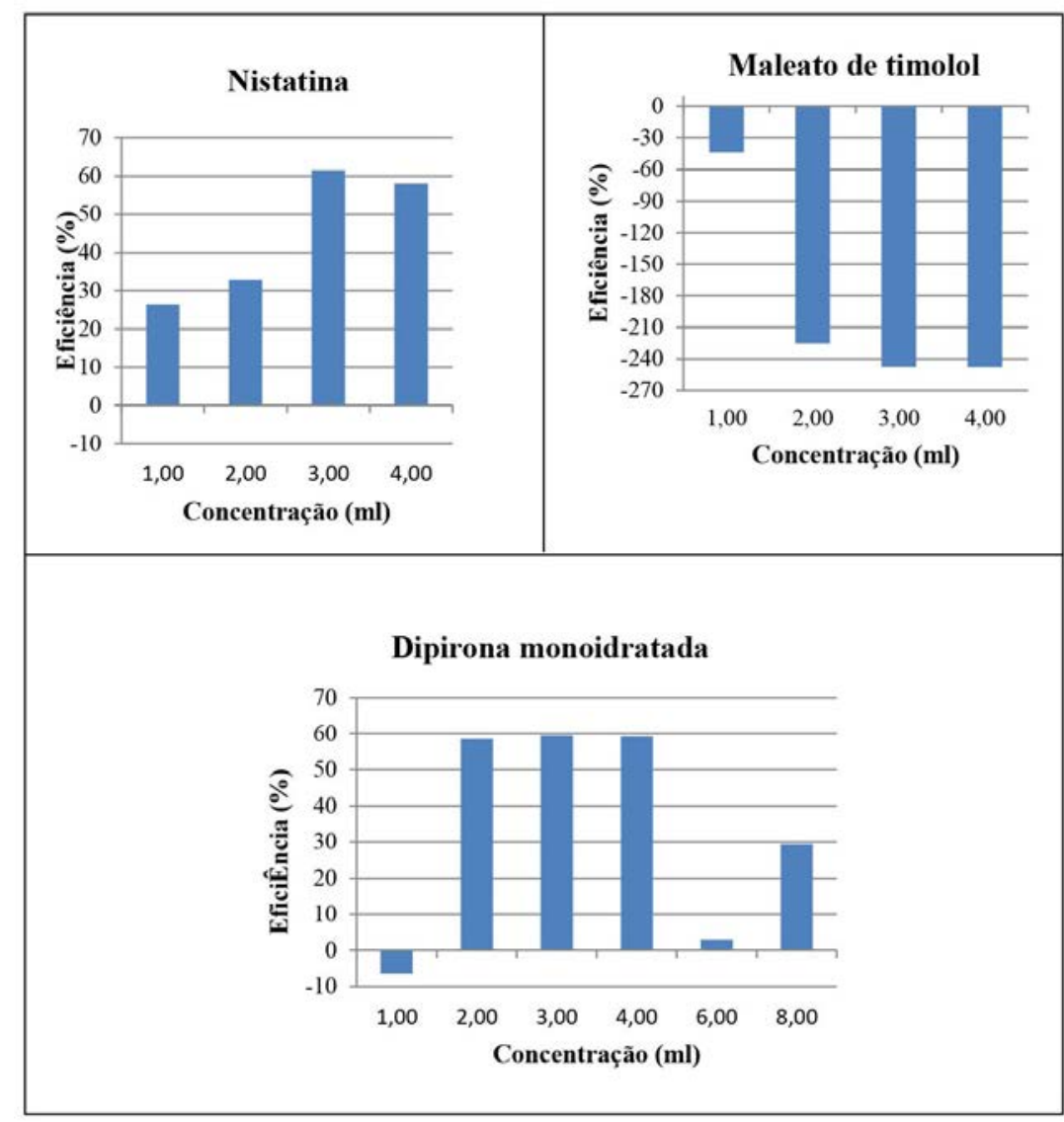

Fonte: Elaboração própria (2019).

\section{Conclusão}

A corrosão em materiais metálicos é um problema que aflige diferentes segmentos da indústria, sendo, em muitos casos, inevitável. A busca por métodos que inibam ou desacelerem o processo de corrosão deve ser estudada constantemente, buscando a utilização de insumos eficientes, abundantes e, também, ecológicos. Uma variedade de fármacos vencidos, em seu estado líquido ou sólido, e que são descartados possuem eficiência contra o processo de corrosão em materiais metálicos. Este trabalho teve como objetivo analisar a eficiência de diferentes fármacos vencidos.

Através dos resultados de TPC e cálculo da eficiência de cada fármaco, percebe-se que a nistatina, dipirona monoidratada e o cloridrato de tipoclidina atingiram níveis bastante satisfatórios de eficiência contra a corrosão em diferentes concentrações. Já os fármacos maleato de timolol, pilem levonorgestrel e apresolina, independente da concentração utilizada, apresentaram efeito contrário, não sendo considerados inibidores de corrosão. Os resultados mostram uma tendência do uso dos fármacos contra o processo de corrosão, devendo ser realizado novos experimentos para confirmar estatisticamente esses resultados, de modo que seja possível afirmar a eficiência ou não dos fármacos como inibidores de corrosão em materiais metálicos. 
Dentre os inibidores, a tendência que os fármacos cloridrato de tipoclidina, dipirona monoidratada e nistatina possam ser utilizados como inibidores de corrosão, uma vez que apresentaram eficiência superior a $66 \%$ em alguns casos. Outros estudos devem ser realizados com esses três fármacos a fim de verificar a concentração ideal de cada fármaco no processo contra a corrosão, além de analisar o mecanismo químico que ocorre para inibir o processo de corrosão. Convém lembrar que as considerações apresentadas foram formuladas em testes experimentais, o que pode ser o motivo da ineficiência da dipirona monoidratada quando utilizado $1 \mathrm{ml}$.

\section{Referências}

ABRACO. Inibidores de Corrosão: confiabilidade e redução de custos. Revista Corrosão e Proteção, Rio de Janeiro, n. 14. p. 9-12, 2007. Disponível em: http://www.abraco.org.br/src/uploads/2016/01/Revistacorrosao-e-protecao-14.pdf. Acesso em: 12 set. 2019.

CALLISTER, W. D. Ciência e engenharia de materiais: uma introdução. 7. ed. Rio de Janeiro: LTC, 2008.

CERQUEIRA, S. P. et al. Análise de fármacos como inibidores de corrosão em materiais metálicos. Trabalho de Conclusão de Curso (Graduação) - Instituto de Ciência, Engenharia e Tecnologia UFVJM, Teófilo Otoni, 2018.

CLORIDRATO DE TIPOCLIDINA: comprimidos. Responsável técnico RonoelCaza de Dio. Hortolândia: EMS, 2013. 1 bula de remédio.

DIGOXINA: comprimidos. Responsável técnico Giovana Bettoni. Anápolis: Vitamedic, 2016. 1 bula de remédio.

FERNANDES, C. E. et al. Utilização de fármacos vencidos como inibidores de corrosão em aço carbono A36 e HCl 1M. In: ENCONTRO TÉCNICO DE MATERIAIS E QUÍMICA, 9., Rio de Janeiro, 2017. Anais [...]. Disponível em: https://www.marinha.mil.br/ipqm/sites/www.marinha.mil.br.ipqm/files/etmq/34.pdf. Acesso em: 12 set. 2019.

FRAUCHES-SANTOS, C. et al A corrosão e os agentes anticorrosivos. Revista virtual de química, 2014. v. 6. p. 293-309. Disponível em http://rvq.sbq.org.br/imagebank/pdf/v6n2a09.pdf. Acesso em: 10 ago. 2018.

KENDIG, M.; SCULLY, J. Basic aspects of electrochemical impedance application for the life prediction of organic coatings on metals. Corrosion, [s. I.], v. 6, n. 1, jan. 1990. Disponível em: https://www. corrosionjournal.org/doi/pdf/10.5006/1.3585061. Acesso em: 01 jun. 2019.

MORA, N. D.; SIHVENGER, J. C. (comp.). Apostila de Química Geral. Foz do Iguaçu, 2006. Disponível em: http://www.foz.unioeste.br/ lamat/downquimica.html. Acesso em: 15 set. 2018.

NASCIMENTO, A. M. et al. Comportamento corrosivo de aços inoxidáveis utilizados como revestimentos em refinarias de petróleo. Revista Tecnológica, Fortaleza, v. 25, n. 1, p. 65-73, jun. 2004.

PARANHOS, C. H. V. et al. Avaliação de uma molécula orgânica derivada do Triazol como inibidor de corrosão em meio ácido de $\mathrm{HCl} 1 \mathrm{M}$. In: ENCONTRO TÉCNICO DE MATERIAIS E QUÍMICA, 9., Rio de Janeiro, 2017. Anais [...]. Disponível em: https://www.marinha.mil.br/ipqm/sites/www.marinha.mil.br.ipqm/ files/etmq/57.pdf. Acesso em: 01 set. 2019.

LI, S. L. et al. Some aspects of quantum chemical calculations for the study of Schiff base corrosion inhibitors on copper in $\mathrm{NaCl}$ solutions. Corrosion Science, China, v. 1, jan. 1999. Disponível em: https:// www.marinha.mil.br/ipqm/sites/www.marinha.mil.br.ipqm/files/etmq/57.pdf. Acesso em: 01 dez. 2019. 


\section{Sobre os autores}

\section{Matheus da Silva Lages}

Mestre em Engenharia mecânica pelo Programa de Pós-Graduação em Engenharia Mecânica da Universidade Federal de Minas Gerais. Graduado em Engenharia mecânica pela Universidade Federal dos Vales do Jequitinhonha e Mucuri. Bacharel em Ciência e Tecnologia pela Universidade Federal dos Vales do Jequitinhonha e Mucuri. Professor temporário na Universidade Federal dos Vales do Jequitinhonha e Mucuri, e professor na Faculdade Presidente Antônio Carlos. Tem experiência em processos de fabricação, tecnologia dos materiais e estudo de sólidos.

\section{Carlos Alexandre Teixeira de Matos}

Graduando em Ciência e Tecnologia pela Universidade Federal dos Vales do Jequitinhonha e Mucuri - campus Mucuri (UFVJM).

\section{Cristiano Batista Rodrigues}

Graduando em Ciência e Tecnologia pela Universidade Federal dos Vales do Jequitinhonha e Mucuri - campus Mucuri (UFVJM).

\section{Ellen Pires Saigg}

Graduanda em Ciência e Tecnologia pela Universidade Federal dos Vales do Jequitinhonha e Mucuri - campus Mucuri (UFVJM).

\section{Karine Silva}

Bacharel em Ciências e Tecnologia pela Universidade Federal dos Vales do Jequitinhonha e Mucuri (2018). Atualmente, é aluna do curso de Engenharia civil na Universidade Federal dos Vales do Jequitinhonha e Mucuri - campus Mucuri. Tem experiência em monitoria na disciplina Ciência e tecnologia dos materiais.

Recebido em: 02.07.2019

Aceito em: 13.11 .2019 